\title{
CVD HAFNIUM DIBORIDE AS A CONTACT MATERIAL FOR NANOELECTROMECHANICAL SWITCHES \\ W.S. Lee ${ }^{l^{*}}$, A.N. Cloud ${ }^{2}$, J. Provine ${ }^{l}$, N. Tayebi ${ }^{l}$, R. Parsa ${ }^{1}$, S. Mitra ${ }^{l}$, H.-S. P. Wong ${ }^{l}$, J. R. Abelson, ${ }^{2}$ and R.T. Howe ${ }^{l}$ \\ ${ }^{1}$ Electrical Engineering Department, Stanford University, Stanford, California, USA \\ ${ }^{2}$ Department of Materials Science and Engineering, University of Illinois at Urbana-Champaign, Urbana, Illinois, USA
}

\section{ABSTRACT}

This paper presents the first usage of hafnium diboride $\left(\mathrm{HfB}_{2}\right)$ deposited via chemical vapor deposition as the contact material for nanoelectromechanical (NEM) switches. $\mathrm{HfB}_{2}$ is an excellent candidate for NEM switches due to its reasonable conductivity, high hardness, and high melting point. $\mathrm{HfB}_{2}$ was deposited conformally at $250^{\circ} \mathrm{C}$ onto the sidewalls of laterally actuated, polysilicon switches. $\mathrm{HfB}_{2}$ coated switches showed sharp switching characteristics when tested in a nitrogen ambient. The contact resistance was initially $29.6 \mathrm{M} \Omega$ but decreased to $1.43 \mathrm{k} \Omega$ by adding a dilute HF dip to remove the surface oxide.

\section{INTRODUCTION}

Complementary metal-oxide-semiconductor (CMOS) scaling has resulted in the mass production and proliferation of microelectronics. Recently, power constraints have become a limiting factor to scaling. Scaling the supply voltage requires simultaneous scaling of the threshold voltage to avoid incurring a delay penalty [1]. Threshold voltage scaling, however, leads to an exponential increase in leakage current. A further reduction in CMOS power consumption requires a device that does not suffer from these same scaling limits such as the nanoelectromechanical (NEM) switch. The NEM switch has zero leakage current, highly scalable actuation voltages, and very sharp switching characteristics $[2,3]$.

A three terminal NEM switch consists of a movable beam electrode, a fixed gate electrode, and a fixed drain electrode. Initially, the gate-to-beam voltage, $V_{G B}$, is zero, and the beam is electrically isolated from the drain so the drain-to-beam current, $I_{D B}$, is zero. As $V_{G B}$ increases, the electrostatic force is balanced by the elastic force of the beam. As $V_{G B}$ continues to increase, the beam can no longer balance the electrostatic force so the beam pulls into the drain, and current flows between the drain and beam. This voltage is called the pull-in voltage, $V_{P I}$, and the switch is in the on-state. As $V_{G B}$ decreases, the electrostatic force also decreases. Due to the reduced gap between the beam and gate in the on state, $V_{G B}$ must decrease below $V_{P I}$ for the beam to pull-out. This voltage is called the pull-out voltage, $V_{P O}$. A five terminal NEM switch has an additional gate and drain electrode that enables bi-directional switching.

Unfortunately, NEM switches suffer from a long mechanical delay. Hybrid systems that combine CMOS with NEM switches have been proposed that take advantage of the low power characteristics of NEM switches while mitigating their long switching delay. Examples include FPGA routing [4], CMOS power gating [5], and SRAM cells [6]. The FPGA routing application showed substantial power and area reductions without a delay penalty by utilizing switches with $2 \mathrm{k} \Omega$ contact resistances [7]. This contact resistance requirement is much higher than the sub- $1 \Omega$ resistances required by RF MEM switch applications [8] and enables the use of harder, more robust contact materials.

We investigate hafnium diboride $\left(\mathrm{HfB}_{2}\right)$ as a contact material for NEM switches. $\mathrm{HfB}_{2}$ is a conductive ceramic with a hardness, elastic modulus, and melting point of $20 \mathrm{GPa}, 400 \mathrm{GPa}$, and $3250^{\circ} \mathrm{C}$
[9], respectively, that can be deposited via chemical vapor deposition (CVD) at back-end-of-line compatible temperatures. Since the deposited film is very conformal, it can be used to coat trenches and form sidewalls [9]. $\mathrm{HfB}_{2}$ has been shown to have higher scratch resistance with a lower coefficient of friction than titanium nitride [10] and has been used as a protective coating on a silicon tip showing negligible wear after $5 \times 10^{9} \mu \mathrm{m}$ of sliding [11]. Hard coatings are desirable for NEM switches because they can suppress plastic deformation of asperities, which leads to variations in contact area, contact resistance, and surface forces.

\section{CONTACT MECHANICS}

NEM switches are actuated either laterally or vertically. We characterize $\mathrm{HfB}_{2}$ with lateral NEM switches because the fabrication process requires a single $\mathrm{HfB}_{2}$ deposition and two mask layers. The fabrication will be further described in the next section. The shape of the contacting surface between the beam and the drain is defined by the sidewall roughness of the polysilicon reactive ion etch. We approximate the contacting surface as a single asperity with a radius, $r$, which is on the order of $10 \mathrm{~nm}$. The contact asperity radius contributes to both the Maxwell resistance, $R_{M}$, and Sharvin resistance, $R_{S}$, components of the total contact resistance, $R_{C}$. They originate from electron lattice scattering and electron boundary scattering, respectively. For a round asperity that undergoes elastic deformation, $R_{C}$ is given by $[12,13]$

$$
R_{C}=\gamma\left(\frac{\lambda}{r}\right) R_{M}+R_{S}=\frac{1+0.83\left(\frac{\lambda}{r}\right)}{1+1.33\left(\frac{\lambda}{r}\right)} \frac{\rho}{2 r}+\frac{4 \rho \lambda}{3 \pi r^{2}}
$$

where $\rho$ is the resistivity of the material, $\lambda$ is the electron mean free path, and $\gamma$ is a fitting function used to transition between the Maxwell and Sharvin resistance regimes. The resistivity of $\mathrm{HfB}_{2}$ was measured to be $303 \mu \Omega-\mathrm{cm}$. Since $\lambda$ is unknown for $\mathrm{HfB}_{2}$, we estimate the contact resistance for two cases. The first case is when the contact asperity is much larger than the electron mean free path (i.e., $\lambda / r \approx 0$ ). The second case is when the contact asperity is roughly the same size as the electron mean free path (i.e., $\lambda / r \approx 1$ ). Figure 1 shows the contact resistance for each of these cases for contact radii ranging from $1 \mathrm{~nm}$ to $50 \mathrm{~nm}$. For a $10 \mathrm{~nm}$ radius, the estimated contact resistance is $150 \Omega$ and $250 \Omega$, for $\lambda / r=0$ and $\lambda / r$ $=1$, respectively. These estimated contact resistances are only based on the contact geometry and do not account for any insulating layer that may appear on the surface of the contact. The measured contact resistance will be higher than these values if there is an oxide or contamination layer on the surface of the contact.

\section{DESIGN AND FABRICATION}

The lateral switch fabrication process flow initially follows the methods presented at Hilton Head 2010 [14] where a $1 \mu \mathrm{m}$ insitu doped polysilicon structural layer is deposited on a $2 \mu \mathrm{m}$ LTO sacrificial layer and annealed. The polysilicon layer is patterned using a photoresist mask exposed with an i-line stepper. The 


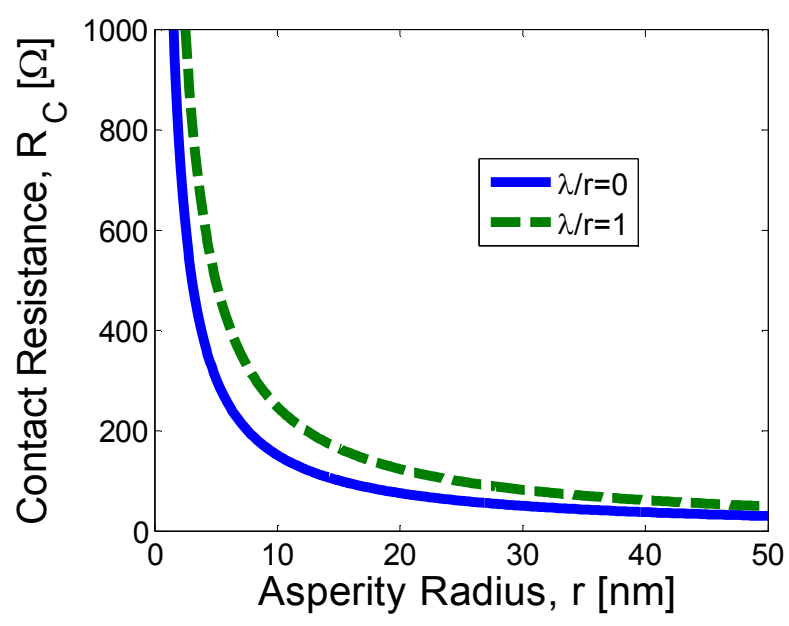

Figure 1: Contact resistance, $R_{C}$, for $\mathrm{HfB}_{2}$ as a function of contact radius for $\lambda / r \approx 0$ (i.e., contact asperity radius is much larger than the electron mean free path) and $\lambda / r \approx 1$ (i.e., the contact asperity radius is approximately the same size as the electron mean free path).

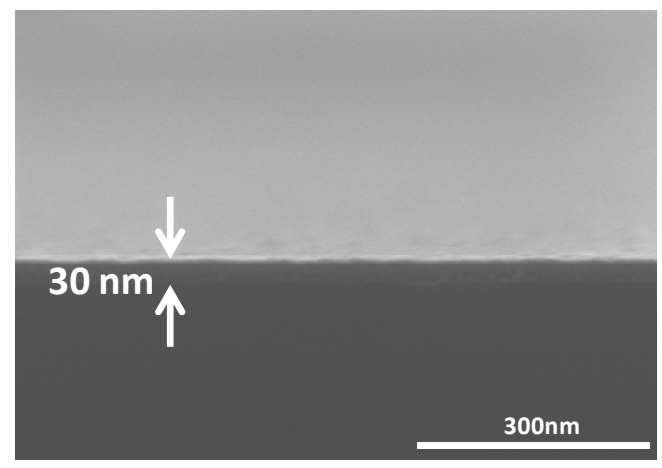

Figure 2: $30 \mathrm{~nm}$ of $\mathrm{HfB}_{2}$ film with subnanometer surface roughness deposited on crystalline silicon by chemical vapor deposition.

pattern is transferred to the polysilicon layer using a reactive ion etch (RIE) (12mTorr, $25 \mathrm{sccm} \mathrm{Cl}_{2}, 140 \mathrm{sccm} \mathrm{HBr}, 3 \mathrm{sccm} \mathrm{O}$ ).

Instead of coating the devices with sputtered platinum as in [14], the devices are coated in $\mathrm{CVD} \mathrm{HfB}_{2}$ at $250^{\circ} \mathrm{C}$ using $\mathrm{Hf}\left(\mathrm{BH}_{4}\right)_{4}$ precursor in an ultra high vacuum chamber as described in [15]. Figure 2 shows an SEM cross section of a typical $30 \mathrm{~nm}$ film deposited on a silicon substrate. A contact aligner is used to define the pads for the devices. The $\mathrm{HfB}_{2}$ is etched using a directional, chlorine-based RIE (5mTorr, 40sccm $\mathrm{BCl}_{3}, 10 \mathrm{sccm} \mathrm{Cl}_{2}, 10 \mathrm{sccm}$ $\mathrm{Ar})$ that removes the $\mathrm{HfB}_{2}$ from the exposed lateral surfaces and electrically isolates the various terminals. A continuous layer of $\mathrm{HfB}_{2}$ remains on the device sidewalls and on the photoresist protected pads of the devices. The photoresist is stripped using an $\mathrm{O}_{2}$ plasma followed by a 10 minute acetone soak and isopropanol rinse.

The switches are released by etching the LTO for 15 minutes in a 5:3 mixture of buffered oxide etch $\left(6\right.$ parts $40 \% \mathrm{NH}_{4} \mathrm{~F}$ to 1 part $49 \% \mathrm{HF}$ ) to glycerin, which obtains a good etch selectivity between the LTO and the $\mathrm{HfB}_{2}$. The switches were dried after the BOE/glycerin etch using a critical point dryer (CPD) to avoid stiction. The final released device is shown in Figure 3. The zoomed-in view of the contacting region in Figure 3 shows the

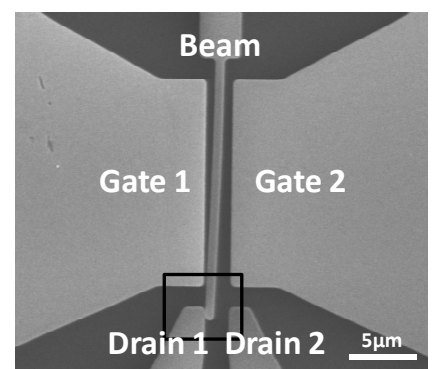

(a)

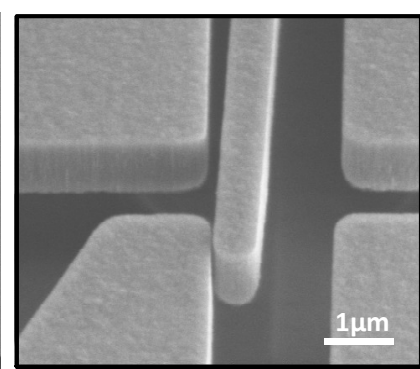

(b)
Figure 3: (a) $\mathrm{HfB}_{2}$ coated relay that is actuated under SEM; (b) zoomed in view of the contact region.

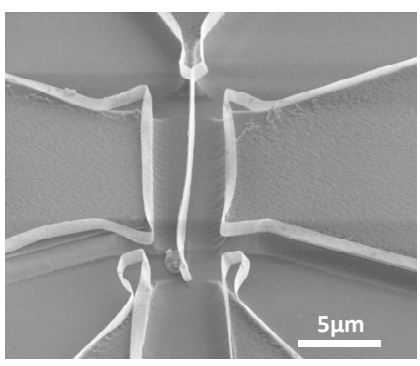

(a)

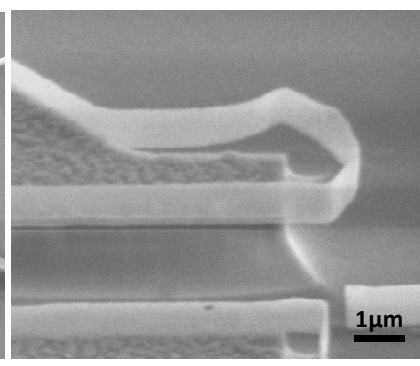

(b)
Figure 4: (a) Zoomed-out view of an $\mathrm{HfB}_{2}$ coated switch that was placed in $\mathrm{XeF}_{2}$ to remove the polysilicon and confirm the presence of $\mathrm{HfB}_{2}$. (b) Zoomed-in view that shows very smooth $\mathrm{HfB}_{2}$ sidewalls.

high conformality of the $\mathrm{HfB}_{2}$ coating. The sidewall surface roughness is equivalent to the bare polysilicon devices and is much smoother than the sputtered platinum coated devices [14]. The presence of $\mathrm{HfB}_{2}$ on the sidewalls was confirmed by etching a device in $\mathrm{XeF}_{2}$ that removed the polysilicon layer without attacking the $\mathrm{HfB}_{2}$ sidewalls as shown in Figure 4.

\section{RESULTS}

The switches were tested in a nitrogen glovebox at room temperature using a Keithley 4200 parameter analyzer. The drainbeam voltage, $V_{D B}$, was set to $1 \mathrm{~V}$, the beam voltage, $V_{B}$, was set to $0 \mathrm{~V}$, and the current compliance was set to $100 \mathrm{nA}$ to protect the contact from welding and Joule heating. $V_{G B}$ was swept from $0 \mathrm{~V}$ to $60 \mathrm{~V}$, and no drain current was measured with the $1 \mathrm{~V} V_{D B}$. The drain-beam voltage was then increased to $5 \mathrm{~V}$, the $V_{G B}$ sweep was repeated, and the current reached the 100nA compliance with sharp switching characteristics as shown in (Figure 5a). To maintain the current compliance, the parameter analyzer adjusts the voltage on both the beam and drain electrodes. By measuring the voltages required to maintain the current compliance, the voltage across the contact and the contact resistance can be calculated. For these initial devices, the contact resistance was $29.6 \mathrm{M} \Omega$, which is much higher than the predicted contact resistance of $250 \Omega$. On the subsequent voltage sweep, the current compliance was increased to $1 \mu \mathrm{A}$ (Figure $5 \mathrm{~b}$ ) while maintaining the $V_{D B}$ at $5 \mathrm{~V}$. The contact resistance decreased to $171 \mathrm{k} \Omega$. For the third voltage sweep, $V_{D B}$ voltage was decreased to $1 \mathrm{~V}$ (Figure $5 \mathrm{c}$ ), the $1 \mu \mathrm{A}$ current compliance was still reached, and the contact resistance decreased to $8.9 \mathrm{k} \Omega$. By passing $1 \mu \mathrm{A}$ of current through the contact, the contact resistance decreased from $29.6 \mathrm{M} \Omega$ to $8.9 \mathrm{k} \Omega$ while the minimum voltage across the contact decreased from $3.0 \mathrm{~V}$ to 


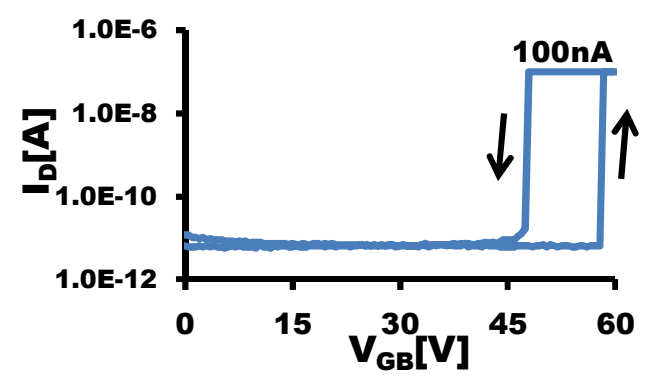

(a)

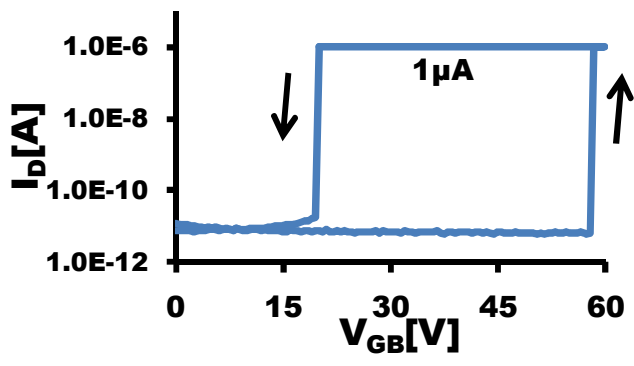

(b)

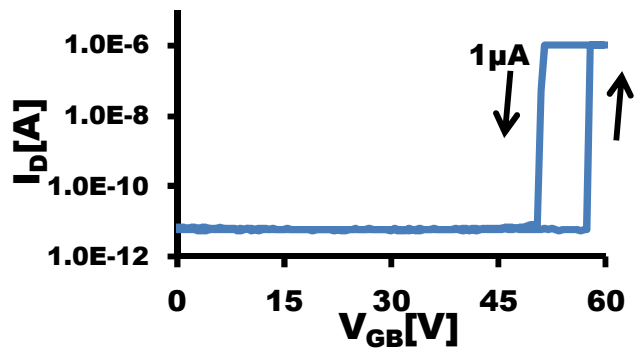

(c)

Figure 5 - (a) Initial IV sweep of $\mathrm{HfB}_{2}$ coated relay that requires high drain voltage to breakdown insulating film $\left(V_{D B}=5 \mathrm{~V}\right.$, Current Compliance=100nA). (b) Subsequent IV sweep with increased current compliance $\left(V_{D B}=5 \mathrm{~V}\right.$, Current Compliance $\left.=1 \mu \mathrm{A}\right)$. (c) Subsequent sweep with decreased drain voltage with the higher current maintained $\left(V_{D B}=1 \mathrm{~V}\right.$, Current Compliance $\left.=1 \mu \mathrm{A}\right)$. Insulating film broken down at contact by high voltage and high current. Actual $V_{D B}$ were less than the setpoint to maintain current compliance.

$8.9 \mathrm{mV}$. These results were indicative of an insulating surface film that experienced breakdown with sufficient current flow.

The $\mathrm{HfB}_{2}$ surface modifications were further characterized by performing a voltage sweep on an unpatterned $\mathrm{HfB}_{2}$ film. The current flow was initially very small. When the applied voltage was $2.8 \mathrm{~V}$, breakdown of the surface was observed, and the current increased significantly (Figure 6a). A second voltage sweep showed only resistive current flow without any breakdown voltage. These results matched the device results showing where the initial contact voltage was measured to be $3.0 \mathrm{~V}$ to observe current flow.

To determine the source of the breakdown voltage, X-ray photoelectron spectroscopy (XPS) was performed on a sample of $\mathrm{HfB}_{2}$ that had been exposed to air for three months. The analysis confirmed the presence of an oxide layer on the surface. As shown in Figure $7 \mathrm{a}$, approximately $80 \%$ of the Hf signal and $50 \%$ of the B

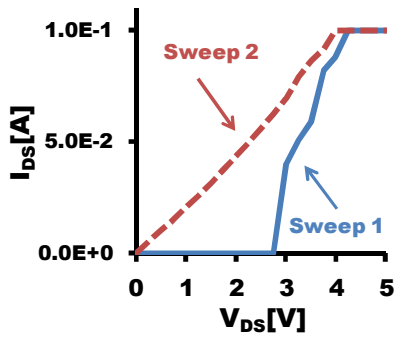

(a)

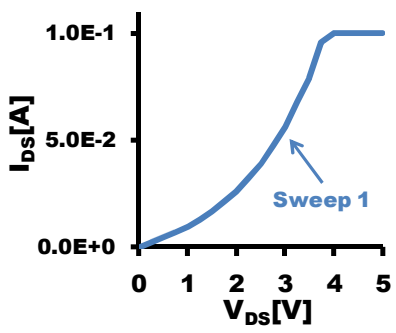

(b)
Figure 6-(a) IV characteristics of $\mathrm{HfB}_{2}$ film without HF dip and (b) with $30 \mathrm{~s}$ dip in 50:1 HF. Two probes were placed on an $\mathrm{HfB}_{2}$ pad, and the voltage between them was swept.

(a)
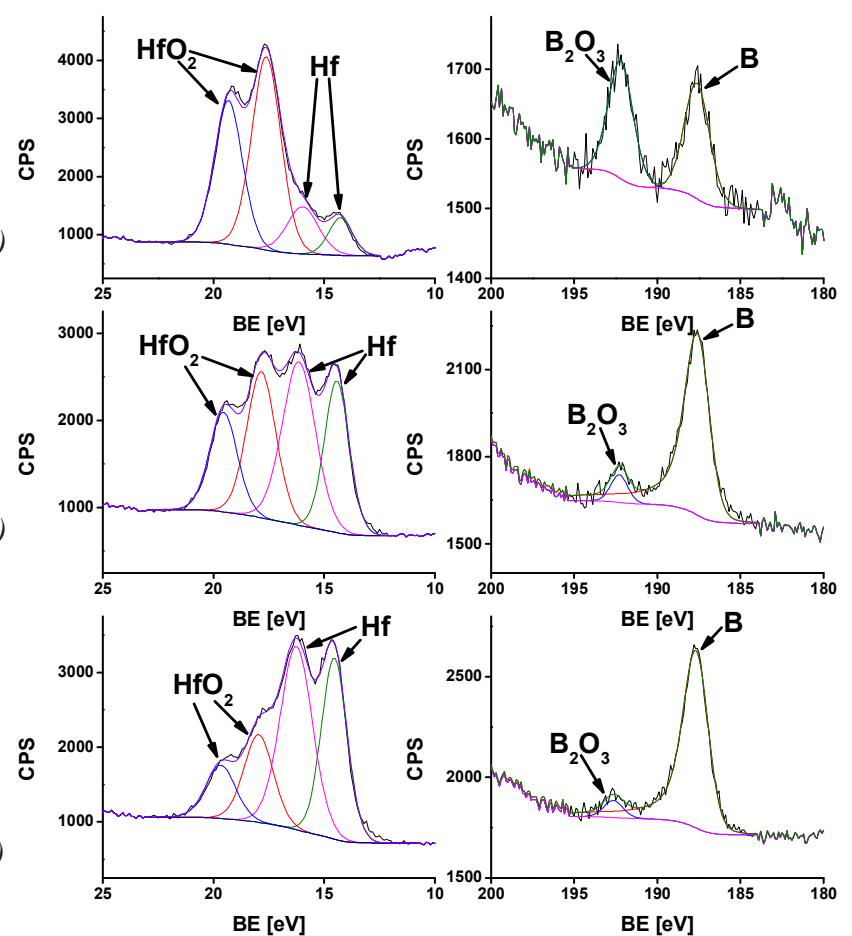

Figure 7 - (a) XPS spectra of a $\mathrm{HfB}_{2}$ film that was exposed to air for 3 months, (middle) Same film after 15 minute etch in glycerin/BOE solution, (bottom) Same film after 15 minute etch in glycerin/BOE and 30s dip in 50:1 HF. The oxygen content of the surface layer is significantly reduced by the etching steps.

signal have binding energy peaks indicative of oxides. The fabricated switches are exposed to a 15 minute etch in the $\mathrm{BOE} / \mathrm{glycerin}$ solution to release the devices that could also affect the surface oxide layer. Therefore, the $\mathrm{HfB}_{2}$ sample was exposed to this same solution for 15 minutes and reanalyzed under XPS. The oxygen content was significantly reduced as shown in Figure $7 \mathrm{~b}$, but it was still present. To further reduce the oxide layer, a 30s dip in 50:1 HF following the BOE/glycerin etch was added. The oxygen content continued to decrease as shown in Figure 7c. The reduced oxygen content is closer to the less than $1 \%$ oxygen content found in the as-deposited film [9]. Based on the XPS results, a $60 \mathrm{~s}$ dip in $50: 1 \mathrm{HF}$ was added to the release process between the BOE/glycerin step and the CPD step. After the CPD step, the samples are immediately stored in nitrogen. The two probe test was repeated and the breakdown was eliminated (Figure $6 b)$. 


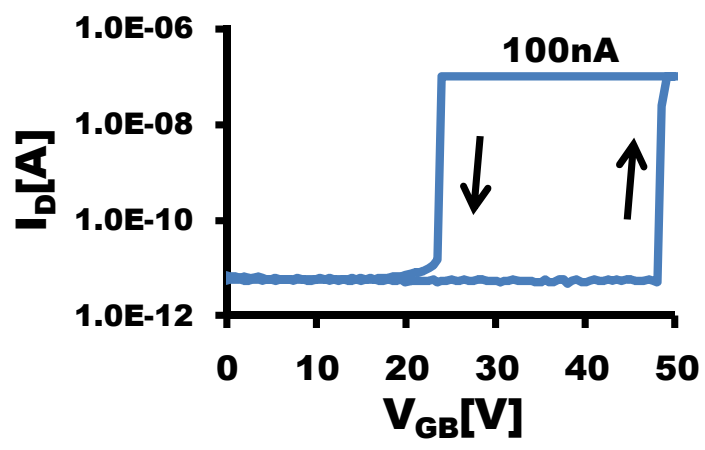

Figure $8-I V$ characteristics of $\mathrm{HfB}_{2}$ coated relay treated with $60 \mathrm{~s}$ etch in 50:1 HF. Required drain voltage is reduced $\left(V_{D B}=1 \mathrm{~V}\right)$ without requiring an initial high drain voltage sweep.

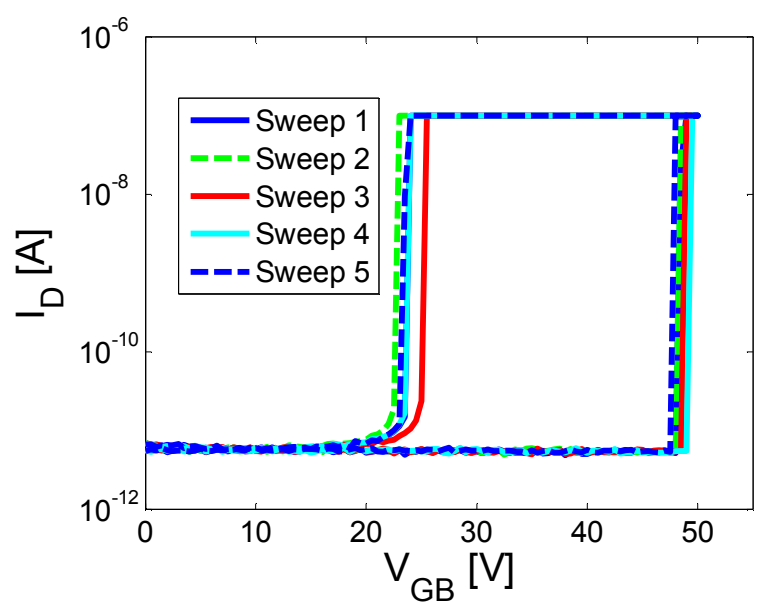

Figure 9 - Five cycles of $\mathrm{HfB}_{2}$ coated switch with $V_{D B}$ set to $1 \mathrm{~V}$ with 100nA current compliance. The contact resistance was measured to be $1.43 \mathrm{k} \Omega$ based on the measured drain-to-beam voltage when actuated.

With the added dilute HF dip, the 100nA current compliance was reached with a $1 \mathrm{~V}$ drain bias without requiring high current to breakdown an insulating film (Figure 8). The measured voltage across the contact was $143 \mu \mathrm{V}$ corresponding to a measured contact resistance of $1.43 \mathrm{k} \Omega$. The switch showed correct operation over five cycles (Figure 9).

\section{CONCLUSION}

$\mathrm{HfB}_{2}$ coated polysilcon switches were fabricated and tested. The switches initially had a contact resistance of $29.6 \mathrm{M} \Omega$, which was attributed to the presence of a surface oxide. By removing this oxide, the contact resistance decreased to $1.43 \mathrm{k} \Omega$, which is close to the $250 \Omega$ calculated contact resistance. The measured contact resistance may be higher due to either the contacting asperity being smaller than the estimated $10 \mathrm{~nm}$ or due to the presence of a thin insulating film on the surface of the contact.

These preliminary results show that $\mathrm{HfB}_{2}$ is a promising NEM switch contact material, but further investigation into long term cycling and device statistics are required. Furthermore, future $\mathrm{HfB}_{2}$ switches should be encapsulated in an oxygen free ambient to prevent oxidation.

\section{ACKNOWLEDGMENTS}

Fabrication work performed at the Stanford Nanofabrication Facility (member of the National Nanotechnology Infrastructure Network) supported by the NSF under Grant ECS-9731293. $\mathrm{HfB}_{2}$ film deposition took place at the University of Illinois at UrbanaChampaign. Materials characterization was carried out in part in the Frederick Seitz Materials Research Laboratory Central Facilities, University of Illinois, which is partially supported by the U. S. Department of Energy under grants DEFG02-07ER46453 and DE-FG02-07ER46471. The work was supported by the DARPA MTO program "Nano Electro Mechanical Computers (NEMS)" (Contract number: NBCH 1090002, Program manager: Dr. T. Akinwande).

\section{REFERENCES}

[1] V. Kursen et al., Multi-Voltage CMOS Circuit Design, John Wiley \& Sons, Ltd., England, 2006.

[2] F. Chen et al., "Demonstration of Integrated Micro-ElectroMechanical Switch Circuits for VLSI Applications", IEEE ISSCC 2010 pp. 150-152.

[3] K. Akarvardar et al., "Design Considerations for Complementary Nanoelectromechanical Logic Gates", IEDM 2007 pp. 299-302.

[4] Y. Zhou et al., "Low Power FPGA Design Using Hybrid CMOS-NEMS Approach", Proc. Intl. Symp. Low Power Electronics and Design, 2007, pp. 14-19.

[5] H. Fariborzi et al., "Analysis and Demonstration of MEMRelay Power Gating”, Custom Integrated Circuits Conference (CICC) (2010) pp. 1-4

[6] S. Chong et al., "Nanoelectromechanical (NEM) Relays Integrated with CMOS SRAM for Improved Stability and Low Leakage", ICCAD 2009, pp. 478-484.

[7] C. Chen et al., "Nano-Electro-Mechanical relays for FPGA Routing: Experimental Demonstration and a Design Technique", IEEE/ACM Design Automation and Test in Europe, Dresden, Germany, March 2012.

[8] G. Rebeiz, RF MEMS: Theory, Design, and Technology, John Wiley \& Sons, Ltd., England, 2004.

[9] S. Jayaraman et al., "HfB ${ }_{2}$ and Hf-B-N Hard Coatings by Chemical Vapor Deposition", Surface and Coatings Technology, 200, 22-23 (2006).

[10] A. Chatterjee et al., "Nanoscratch and Nanofriction Behavior of Hafnium Diboride thin films", Wear, 265 (2008).

[11] N. Tayebi et al., "Ultra Hard $\mathrm{HfB}_{2}$ Metallic Tip-Coatings for Ultra-High Density Probe-Based Storage", ACS Nano [Submitted].

[12] B. Nikolic et al., "Electron Transport Through a Circular Constriction", Physical Review B, 60, 6 (1999).

[13] B. Jensen et al., "Effect of Nanoscale Heating on Electrical Transport in RF MEMS Switch Contacts", JMEMS, 14, 5 (2005).

[14] R. Parsa et al., "Composite Polysilicon-Platinum Lateral Nanoelectromechanical Relays", Technical Digest of the 2010 Solid-State Sensor and Actuator Workshop, Hilton Head Isl., SC, 6/6-10/2010, Transducer Research Foundation, Cleveland (2010), pp. 7-10.

[15] S. Jayaraman et al., "Hafnium Diboride Thin Films by Chemical Vapor Deposition from a Single Source Precursor", J. Vac. Sci. Technol. A 23, 6 (2005).

\section{CONTACT}

*W.S. Lee, tel: +1-650-918-7006; wslee@stanford.edu 